\title{
Case history: psychiatric management of adult autism
}

\section{Gregory O'Brien}

$\mathrm{JB}$ is a 31-year-old man who has been described as having borderline intelligence/mild learning disability. (His overall IQ is difficult to assess, but has been estimated as around 70 , or above.) Over the period of his childhood, he was assessed by numerous child psychiatrists and psychologists, all of whom agreed that he had a diagnosis of autism or some related pervasive developmental disorder, although his presentation was always described as 'atypical'. The cause of his developmental disorder has never been established, despite the conventional extensive biological, genetic and other physical investigations (Gillberg, 1990).

In keeping with a diagnosis of autism or related pervasive developmental disorder, he has numerous disabilities (Rutter, 1983). First of all, he has limited useful expressive language. He typically speaks in brief sentences and word groupings and also has some echolalia. It has proven impossible to develop his language skills further, despite extensive input by speech therapy and developmental psychology. In common with many individuals affected by autism, his everyday living is greatly impaired by repetitive movements including hand-flapping, face-slapping and bodyrocking. He commonly eats non-nutrient materials, including faeces. He therefore requires supervision in toileting: a situation which has continued throughout his life, despite extensive efforts to change this. He has a most refractory insistence on routine, particularly concerning dressing, travelling, and his own immediate environment. On the other hand, he has no additional sensory or physical disability, nor any other medical problems. $\mathrm{He}$ is able to dress himself, although he does require constant prompting in this. He is able to feed himself, although due to his pica and his routines, it is often easier for carers to assist him in feeding. He is generally disinterested in other people, although he does now tolerate their company fairly well.

He lived with his parents until his mid-teens, then moved to a further education establishment run by a national charity, and now lives in a local group home. He has been there for some 12 years. The staff group where he lives is fairly adept at the management of disturbed behaviour, and have worked well with him over the years. His current pattern of tolerating other people, although not actually socialising with them, is something of a triumph of recent years. This is regarded by staff as promising. The staff at the house regard JB as an unusual man. Although they do not understand some of his behavioural characteristics, they do accept that he does not react well to efforts to change his complex routines. Also, they find that attempts to communicate with him on emotional or social issues are tolerated poorly. However, they have persistently worked on his emotional communicative skills in recent years. It is to the staff's credit that their efforts to engage him in socialising and emotional communication have not sparked off dramatic or difficult behaviours over the years he has lived in this placement.

JB now presents with a new set of problem behaviours. He has begun to hit one of the men with whom he lives. This has been going on for about two to three months. Initially, it consisted of the occasional trivial punch whenever the man walked past him, but over the past week there have been three occasions when he has attacked him more viciously, and on one occasion drew blood by biting. Initial enquiries with the staff reveal that this aggression does not occur with any other person in the house. The day centre where JB attends has not experienced this problem of late,

Gregory O'Brien is Consultant Psychiatrist in Learning Disabilities at Northgate Hospital, and Clinical Lecturer in the University Department of Psychiatry at Newcastle upon Tyne. His interests include the study of behavioural phenotypes, the psychiatric sequelae of child head injury and particularly the adult outcome of child disabilities such as autism and related pervasive developmental disorders. 
although the staff there have noticed that he has been somewhat prone to tearfulness over the past month or so. They have also noted that he has been generally quick tempered. Further enquiries reveal that general orientation skills, sleep pattern, appetite and other key aspects of life-style and functioning show no recent changes.

Your urgent advice on psychiatric management of this case is required. The leader of the house has indicated that, unless something can be done quickly, he might lose his place there.

\section{Opinion}

Faced with such extreme behavioural change, the first question which might arise is whether to admit this man to some kind of in-patient facility. In a case such as this, the advantages and disadvantages of such a step need to be weighed up very carefully indeed. On the one hand, admission would alleviate the staff care burden, and given his long history of previously settled behaviour, the staff might be happy to have him back should he improve for whatever reason. On the other hand, for someone who is so prone to react to environmental changes as this, admitting him to any inpatient facility will take away the one opportunity to find out just what has been going on. It would certainly not be my first choice to admit this man.

The key to management here is investigation. I would focus my attention in three areas in a case such as this. First of all, repeat physical investigations. If the aggression is indeed as uncharacteristic as described, then a physical and medical problem must be carefully sought. In my experience, this accounts for a minority of cases, but these are so important that it is crucial to rectify them as quickly as possible. Secondly, it is important to look for any additional signs of any mental illness. In this case, I think this is unlikely. All the signs are that although JB is possibly rather more emotionally labile, aggression is focused very much on one person. Also, in other respects he shows no signs of pervasive or persistent substantial behavioural change. This leads us to the third focus of the investigation which concerns the relationship between JB and the man whom he has been assaulting. There are obvious questions to be asked. For example, is JB being provoked deliberately? Are there any signs of any other difficulty between the two people, and if so what is their nature and history?

In cases such as this, often the answer is not immediately obvious. One approach would be to focus on JB, looking carefully at the situation from his point of view, particularly to see whether there is anything which the other party is doing which might be seen as a 'threat' or other intrusive factor into JB's autistic lifestyle. A crucial element of such an analysis of JB's own behaviour would be to consider whether his aggression might be serving him some useful purpose. Common examples of this in autistic adults include aggression which allows the individual to avoid certain activities, especially those entailing personal intimacy or other unwelcome social contact. In addition to an examination of JB's own behaviour, its antecedents and consequences, the problem can be approached at other levels. One option would be to consider the matter as essentially an interactional one between the two men, and to explore how their behaviours and reactions towards each other might be maintaining and promoting problems such as JB's aggression. In this connection, the question of some kind of sexual activity between the two should be borne in mind, although in the present case this would be unlikely (although probably more likely than, for example, mental illness).

Another issue is the reaction of staff and others to JB's aggression. It is quite possible that different staff are adopting different strategies towards him. This is common. Puzzling or worrying behaviour is often met by contrasting and conflicting management styles on the part of different members of staff. What tends to happen is that different people try to think of their own way of dealing with the behaviour, and this results in inconsistencies in management. When one remembers the importance of routine and consistency in respect of autistic people, this can obviously be counter-productive. One possibility which should be borne in mind is that JB has been 'pushed' too quickly in the area of socialising. The latter scenario is encountered not uncommonly in dealing with autistic people, although there is equivocal evidence that it has happened in this case.

A thorough behavioural analysis of problem behaviour such as JB's aggression might entail all of these elements, and more besides. There are numerous techniques and scales available for this purpose. (See Emerson,1995 for update and review.) Broadly speaking, the approaches which might be employed in the present set of problems can be divided into 'event sampling' and 'time sampling'. In event sampling only the occurrence of the identified problem (here, this might be JB's aggression towards the other resident) is required to be recorded, and this can be done in as close detail as deemed appropriate. This would probably be welcomed by staff who have identified the aggression as such a problem, and would therefore 
gain compliance in recording. However, it would yield little insight into other occurrences which might be relevant to the problem behaviour.

Time sampling, in which the occurrence of the index problem and of other identified behaviours in a given time period are recorded, would serve this purpose. Some behavioural analyses involve direct observation. Furthermore, other techniques, including video recording and computer-assisted event recording are popular with some clinicians in the field. Given the time and resource constraints within which most of us work, coupled often with the need for special expertise and training, such approaches are not widespread.

The interventions to be made will of course depend on the insights yielded by behavioural analysis. In fact, when staff become actively involved in such investigations, the manifest behavioural problems often subside. Also, many of the most effective behavioural treatment approaches focus attention away from the identified problem, for example by concentrating on issues such as occupying time appropriately in leisure pursuits or in the acquisition of some new skill.

I would also add a few other points on the immediate management of this case. First of all, just as I would be most reluctant to take the man out of his living situation, similarly I would be reluctant to use any behaviour suppressant medication. However, this is not an absolute veto, depending on further escalation of behaviour, and the results of the immediate investigations. Secondly, it is crucial in managing such cases to remain optimistic and to remind staff of the long history of positive behaviour he has shown, and to particularly emphasise how he has been progressing. In general, the most effective approaches to working with staff groups are those which encourage staff participation, through their increased understanding and knowledge of the problems encountered. (See Yule \& Carr (1987) for further details of working with staff groups in the management of difficult behaviour.)

Finally, whatever interventions prove to be most useful in this case, it would be important to emphasise to JB that this kind of aggressive behaviour is entirely unacceptable. Consistency in his management, and an emphasis that the behaviour is quite unacceptable, will be crucial elements in management of this case.

\section{References}

Emmerson, E. (1995) Challenging Behaviour: Analysis and Intervention in People with Learning Difficulties. Cambridge: Cambridge University Press.

Gillberg, C. (1990) Autism and pervasive developmental disorders. Journal of Child Psychology and Psychiatry, 31, 99120.

Rutter, M. (1983) Cognitive deficits in the pathogenesis of autism. Journal of Child Psychology and Psychiatry, 24,513-532.

Yule, W. \& Carr, J. (1987) Behaviour Modification for People with Mental Handicaps. New York: Croom Helm.

\section{Multiple choice questions}

1. In adults affected by autism:

a Language developmental delay is uncommon

b Individuals commonly tolerate the company of others, if not actively joining in their social activities

c Efforts made by others to encourage socialising may cause behaviour problems

d Aggressive behaviour is rare

e Other mental illnesses do not occur.

2. Investigation of acute behaviour problems in autism:

a Requires admission to an in-patient unit

b In most cases a physical illness is found to be the cause

c Direct observation is required in behavioural analysis

d Time or event sampling might be employed in behavioural analysis

e It is not helpful to involve care staff in behavioural recording.

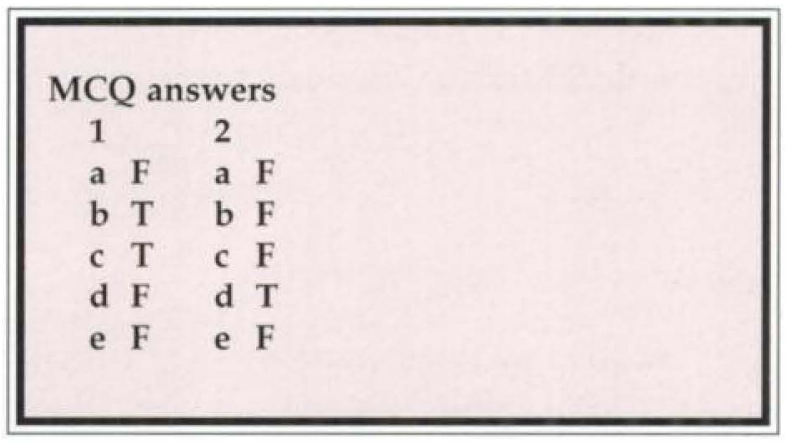

